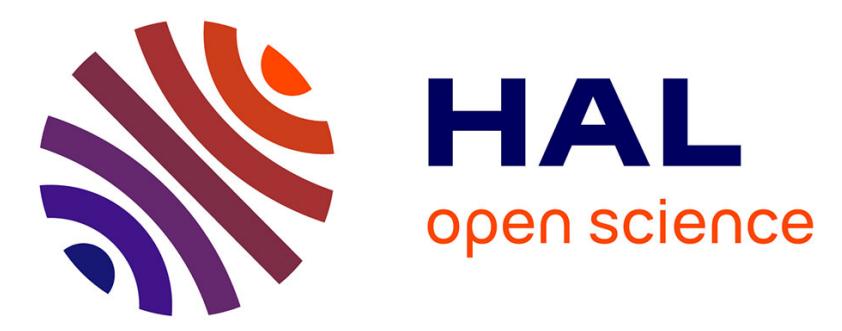

\title{
Durabilité des matériaux composites sous sollicitations hygrothermomécaniques
}

\author{
Frédéric Jacquemin, Bun Eang Sar, Hossein Ramezani Dana, Annick \\ Perronnet, Sylvain Fréour, Pascal Casari
}

\section{- To cite this version:}

Frédéric Jacquemin, Bun Eang Sar, Hossein Ramezani Dana, Annick Perronnet, Sylvain Fréour, et al.. Durabilité des matériaux composites sous sollicitations hygrothermomécaniques. Revue des composites et des matériaux avancés = Journal of Composite and Advanced Materials, 2013, 23 (1), pp.19-34. 10.3166/rcma.23.19-34 . hal-01006756

\section{HAL Id: hal-01006756 https://hal.science/hal-01006756}

Submitted on 21 Jan 2018

HAL is a multi-disciplinary open access archive for the deposit and dissemination of scientific research documents, whether they are published or not. The documents may come from teaching and research institutions in France or abroad, or from public or private research centers.
L'archive ouverte pluridisciplinaire HAL, est destinée au dépôt et à la diffusion de documents scientifiques de niveau recherche, publiés ou non, émanant des établissements d'enseignement et de recherche français ou étrangers, des laboratoires publics ou privés. 


\section{Durabilité des matériaux composites sous sollicitations hygrothermomécaniques}

\section{Frédéric Jacquemin, Bun-Eang Sar, Hossein Ramezani-Dana, Annick Perronnet, Sylvain Fréour, Pascal Casari}

Institut de Recherche en Génie Civil et Mécanique (UMR CNRS 6183)

LUNAM Université - Université de Nantes - Centrale Nantes

CRTT, 37 Boulevard de l'Université

BP 406

F-44602 Saint-Nazaire cedex

frederic.jacquemin@univ-nantes.fr

RÉSUMÉ. L'objectif de cet article est de présenter les points-clés d'une étude de durabilité pour des matériaux composites soumis à un environnement hygrothermomécanique. La démarche permettant de réaliser des essais de vieillissement afin d'identifier le comportement diffusif est présentée. Les effets induits sur le comportement mécanique et les phénomènes de couplages hygrothermomécaniques sont ensuite examinés.

ABSTRACT. The scope of the paper is to present the key points to be addressed in a durability study in the case composite materials in hygrothermomechanical environments. The experimental aging tests allowing the identification of the diffusive behavior arepresented. The induced effects on the mechanical behavior and the hygrothermomechanical coupling phenomenon are also examined.

MOTS-CLÉS: durabilité, comportement diffusif, contraintes internes, approches couplées. KEYWORDS: durability, diffusive behavior, internal stresses, coupling approaches. 


\section{Extended abstract}

Polymer matrix composite structures are often subjected in service to hygrothermal environments (relative humidity and temperature). The constituents of the material can exhibit a heterogeneous hydrophilic nature since polymers absorb moisture whereas most of the inorganic reinforcements are hydrophobic. Two parameters are often used to characterize the moisture diffusion process: the maximum moisture absorption capacity and the diffusion coefficient. However, these parameters can depend on the temperature or relative humidity and in some practical cases on the applied mechanical load.

A Fickian diffusion (governed by the maximum absorption capacity and the diffusion coefficient) is often sufficient for achieving an appropriate simulation of the moisture sorption process occurring in composite materials subjected to ambient relative humidity and temperature. If the moisture sorption presents a more complex behavior, more elaborate approaches can be used. In that context, instead of Fick's model, one can use Langmuir's model (Carter, Kibler, 1978) or the so-called "strong" stress-diffusion coupling models such as the free volume theory or thermodynamically based models.

The moisture sorption can also lead to a plasticization of the constitutive resin, and thus to a decrease of $\mathrm{Tg}$ (glass transition temperature), rigidity and yield stress. As an example, for epoxy resin, the $\mathrm{Tg}$ decrease can reach 30 or $40^{\circ} \mathrm{C}$ for moisture contents around 2 to $3 \%$ (Jedidi, 2005). This phenomenon will be called "weak coupling" as only the mechanical properties depend on the moisture content since the diffusive behavior is independent from the internal mechanical states.

The moisture diffusion experienced by a polymer matrix composite, exposed to a humid environment induces a hygroscopic expansion. The hygroscopic expansion is obviously driven by the expansion coefficients, whose identification is a critical feature in the context of investigating the durability of such structures. The hygroscopic expansion induces internal strain fields yielding internal stresses of two kinds: at a macroscopic scale (i.e. ply scale) as well as at microscopic scale (i.e. in the very constituents of the composite plies). The numerical determination of the induced multiscale mechanical states can be carried out through scale transition models (Jacquemin et al., 2005). Such an approach enables the geometry of the microstructure, the volume fractions of the constituents and the heterogeneous distribution of moisture between the fibers and the matrix to be taken into account.

First of all, the present contribution describes the characterization of the hygoscopic behaviour of glass-fibre reinforced polymer composites, from their kinetics law to the determination of their coefficient of moisture expansion. Then, two coupling models are considered to account for the moisture sorption dependency on the internal mechanical states: a phenomenological approach based on the free volume concept and a more fundamental model, based on a thermodynamic approach. 


\section{Introduction}

Au cours de leur vie en service, les matériaux composites absorbent de l'humidité par le biais de leur matrice polymère. La caractérisation de ce comportement comporte deux aspects. Le premier concerne les mécanismes décrivant la pénétration de l'humidité dans le matériau. Deux paramètres définissent ce processus : la capacité maximale d'absorption et le coefficient de diffusion. Afin de les identifier à l'aide d'une loi de diffusion adéquate, on réalise un suivi gravimétrique lors d'essais de vieillissement. Le second aspect porte sur les effets induits par l'humidité sur la durabilité des structures composites. Notons que les constituants de ces matériaux présentent des coefficients de dilatation hygroscopique hétérogènes (Tsai, 1987). L'expansion hygroscopique est pilotée par ces coefficients de dilatation. La diffusion de l'humidité étant associée à des constantes de temps élevées, il en résulte des gradients de la teneur en eau en fonction de l'épaisseur en régime transitoire (Crank, 1975). Des états de contraintes internes multi-échelles se développent alors. Ces distributions de contraintes dépendent de nombreux facteurs : conditions environnementales, propriétés multi-échelles, microstructure...

La connaissance des contraintes internes est une information capitale pour prédire la durabilité des matériaux composites. Des efforts doivent être réalisés afin de développer des modèles pour prédire les évolutions des états mécaniques multiéchelles durant le processus de diffusion. Les approches présentées dans cet article permettent d'examiner les effets induits par les états mécaniques internes, aux échelles des plis et de ses constituants, sur la cinétique de diffusion. Deux cadres de modélisation sont abordés : l'un phénoménologique, basé sur la théorie du volume libre et l'autre, fondamental, fondé sur une approche thermodynamique.

\section{Comportement hygro-élastique des composites à matrice polymère}

\subsection{Hydrophilie des composites à matrice polymère}

L'hydrophilie d'un composite à matrice polymère est caractérisée par sa capacité maximale d'absorption d'humidité atteinte en régime permanent dans des conditions de température et d'humidité relatives constantes. La capacité maximale d'absorption d'humidité d'un polymère exposé à une humidité relative $\Phi$ satisfait souvent la forme suivante (Loos, Springer, 1979):

$$
\mathrm{M}_{\mathrm{S}}=\mathrm{a} \Phi^{\mathrm{b}}
$$

où $a$ et $b$ sont deux constantes intrinsèques au matériau. D'après Shirrell (1978), l'exposant b est souvent proche de 1 dans le cas de composites renforcés par des fibres de carbone. Les résultats obtenus dans (Pierron et al., 2002) confirment cette évolution linéaire de la capacité maximale d'absorption d'humidité avec l'humidité relative. 
Une deuxième caractéristique fondamentale du comportement diffusif des composites à matrice polymère est la vitesse apparente du processus en régime transitoire. Que la diffusion soit simplement fickienne (Fick, 1855 ; Crank, 1975) ou suive plutôt une cinétique de Langmuir (Carter, Kibler, 1978), cela revient à définir un ou plusieurs coefficients de diffusion de l'humidité. Ces coefficients de diffusion dépendent de la température selon des lois arrhéniennes (Shirrell, 1978; Woo, Piggott, 1987 ; Pierron et al., 2002). Quelques données indiquent que la diffusivité pourrait également dépendre de la teneur en eau (Augl, 1979).

\subsection{Etats mécaniques associés - lois de comportement hygro-élastiques}

\subsubsection{Introduction}

Dans le cas des matériaux composites à base de polymères renforcés, un modèle à deux échelles peut suffire pour décrire le comportement d'un pli composite :

- les propriétés ainsi que les états mécaniques d'un constituant seront notés par l'exposant $\mathrm{i}$. La matrice et le renfort d'un pli composite seront désignés par $\mathrm{m}$ et $\mathrm{r}$. Ces constituants définissent l'échelle «pseudo-macroscopique » du matériau;

- des opérations d'homogénéisation fournissent une estimation du comportement "macroscopique» effectif du pli composite. L'échelle sera représentée par l'exposant I. La présente définition permet de prendre en compte un renforcement uni-directionnel de chacun des plis composites. Le cas de structures renforcées par des fibres courtes ou des tissus, implique un traitement particulier dont un exemple a été décrit dans (Lacoste et al., 2010).

\subsubsection{Lois de comportement hygro-élastiques multiéchelles}

Par analogie avec le cas thermo-élastique, la loi de Hooke multi-échelle hygrothermo-élastique s'écrit (Delasi, Whiteside, 1978) :

$$
\begin{gathered}
\varepsilon^{\mathrm{I}}=\varepsilon_{\mathrm{el}}^{\mathrm{I}}+\varepsilon_{\text {th }}^{\mathrm{I}}+\varepsilon_{\mathrm{hy}}^{\mathrm{I}}=\mathrm{L}^{\mathrm{I}^{-1}}: \sigma^{\mathrm{I}}+\mathbf{M}^{\mathrm{I}} \Delta \mathrm{T}+\beta^{\mathrm{I}} \Delta \mathrm{C}^{\mathrm{I}} \\
\varepsilon^{\mathrm{i}}=\varepsilon_{\mathrm{el}}^{\mathrm{i}}+\varepsilon_{\mathrm{th}}^{\mathrm{i}}+\varepsilon_{\mathrm{hy}}^{\mathrm{i}}=\mathrm{L}^{\mathrm{i}^{-1}}: \sigma^{\mathrm{i}}+\mathbf{M}^{\mathrm{i}} \Delta \mathrm{T}+\boldsymbol{\beta}^{\mathrm{i}} \Delta \mathrm{C}^{\mathrm{i}}
\end{gathered}
$$

où les indices hy, el et th représentent respectivement les parties hygroscopique, purement élastique et thermique du tenseur de la déformation $\boldsymbol{\varepsilon}, \sigma$ est la contrainte, $\Delta \mathrm{C}$ l'incrément de teneur en eau et $\Delta \mathrm{T}$ la variation de température. Les tenseurs $\mathrm{L}$, $\boldsymbol{M}$ et $\boldsymbol{\beta}$ sont la raideur et les coefficients de dilatation thermique ou hygroscopique.

D'après Jacquemin et al. (2005) le coefficient d'expansion hygroscopique effectif d'un composite à fibres hydrophobes satisfait :

$$
\boldsymbol{\beta}^{\mathrm{I}}=\mathrm{v}^{\mathrm{m}} \frac{\Delta \mathrm{C}^{\mathrm{m}}}{\Delta \mathrm{C}^{\mathrm{I}}} \mathbf{L}^{\mathrm{Y}^{-1}}\left(\left(\mathbf{L}^{\alpha}+\mathbf{L}^{\mathrm{I}}: \mathbf{R}^{\mathrm{I}}\right)^{-1}\right)_{a=\mathrm{f}, \mathrm{m}}^{-1}:\left(\mathbf{L}^{\mathrm{m}}+\mathbf{L}^{\mathrm{I}}: \mathbf{R}^{\mathrm{I}}\right)^{-1}: \mathbf{L}^{\mathrm{m}}: \boldsymbol{\beta}^{\mathrm{m}}
$$


où \langle\rangle$_{\alpha=f, m}$ représente la moyenne volumique sur le volume élémentaire représentatif et $\mathrm{v}^{\mathrm{m}}$ la fraction volumique de résine. La détermination pratique de ces moyennes volumiques a fait l'objet de discussions (Fréour et al., 2007 ; 2011).

\section{Caractérisation du comportement diffusif de composites à matrice polymère}

\subsection{Introduction}

Les états mécaniques d'un' matériau composite soumis à un environnement humide dépendent de la cinétique de diffusion d'humidité (Fahmy, Hurt, 1980). Ainsi, la connaissance du coefficient de diffusion associé est indispensable à la modélisation. Les modèles de transition d'échelles permettent d'estimer les coefficients de diffusion effectifs d'un composite unidirectionnel (Shen, Springer, 1976 ; Rayleigh, 1892 ; Shirell, Halpin, 1977; Woo, Pigott, 1988). Dans cette étude, nous allons comparer la diffusivité transverse obtenue expérimentalement aux valeurs prédites par des modèles de transitions d'échelles. Dans cette optique, des composites unidirectionnels contenant différentes fractions volumiques de fibres, ainsi que des échantillons de résine pure ont été élaborés. Leurs coefficients de diffusions transverses ont été identifiés lors d'essais de vieillissement.

\section{2. Élaboration et caractérisation des échantillons}

\subsubsection{Elaboration des échantillons}

Les éprouvettes composites, réalisées par moulage au contact, se composent de fibres de verre de type $\mathrm{E}$ et d'une résine polyester (POLYLITE 420-73), polymérisant à température ambiante. Plusieurs profilés composites, contenant différents taux de fibres $\left(v_{f}\right)$, ainsi qu'un profilé de résine pure, ont été élaborés. Les éprouvettes ont une section de $63 \mathrm{~mm} \times 6 \mathrm{~mm}$ et une longueur de $180 \mathrm{~mm}$.

\subsubsection{Détermination de la fraction volumique de fibres}

La fraction volumique de renforts modifie la valeur du coefficient de diffusion transverse (Kondo, Taki, 1984). Différentes techniques permettent de déterminer cette fraction volumique : l'analyse d'image, l'analyse dimensionnelle, la digestion à l'acide et la calcination (Allred, Hall, 1979 ; Green, 1991 ; Ye et al., 1995). Dans le cadre de la présente étude, la méthode basée sur l'analyse d'image a été appliquée. La section de chaque échantillon a ainsi été couverte par 30 micrographies réalisées à l'aide d'un microscope électronique à balayage (MEB : CARL ZEISS-EVO40).

La figure 1 montre un très bon contraste entre les fibres et la matrice. Ceci permet de déterminer le nombre de pixels associé aux fibres sur chaque 
micrographie et d'obtenir la concentration surfacique de fibres dans l'échantillon. Les résultats obtenus par cette méthode sont présentés dans le tableau 1.

Figure 1. Reconstitution de la section du profilé contenant $53,1 \%$ de fibres de verre à partir de 60 clichés

Tableau 1. Fraction volumique de fibres de verre déterminée par analyse d'image

\begin{tabular}{|c|c|c|c|c|c|c|c|c|}
\hline $\begin{array}{c}\mathrm{N}^{\circ} \text { de } \\
\text { l'échantillon }\end{array}$ & 1 & 2 & 3 & 4 & 5 & 6 & 7 & 8 \\
\hline $\mathrm{v}_{\mathrm{f}}(\%)$ & $\begin{array}{c}41,6 \pm \\
1,7\end{array}$ & $\begin{array}{c}40,6 \pm \\
1,4\end{array}$ & $\begin{array}{c}40,1 \pm \\
1,9\end{array}$ & $\begin{array}{c}45,2 \pm \\
1,3\end{array}$ & $\begin{array}{c}47,2 \pm \\
1,5\end{array}$ & $\begin{array}{c}49,2 \pm \\
1,2\end{array}$ & $\begin{array}{c}52,8 \pm \\
1,4\end{array}$ & $\begin{array}{c}53,4 \\
\pm 1,1\end{array}$ \\
\hline
\end{tabular}

\subsubsection{Vieillissement et identification des paramètres des cinétiques de diffusion}

\subsubsection{Protocole de vieillissement et résultats des mesures}

Des essais de vieillissement ont été effectués dans une enceinte climatique conditionnée en température $\left(23^{\circ} \mathrm{C}\right)$ et en humidité relative $(80 \%)$ afin de caractériser les paramètres de diffusion des échantillons composites et de résine pure. Le suivi gravimétrique a été assuré au moyen de pesées régulières à l'aide d'une balance électronique analytique de résolution $0,1 \mathrm{mg}$.
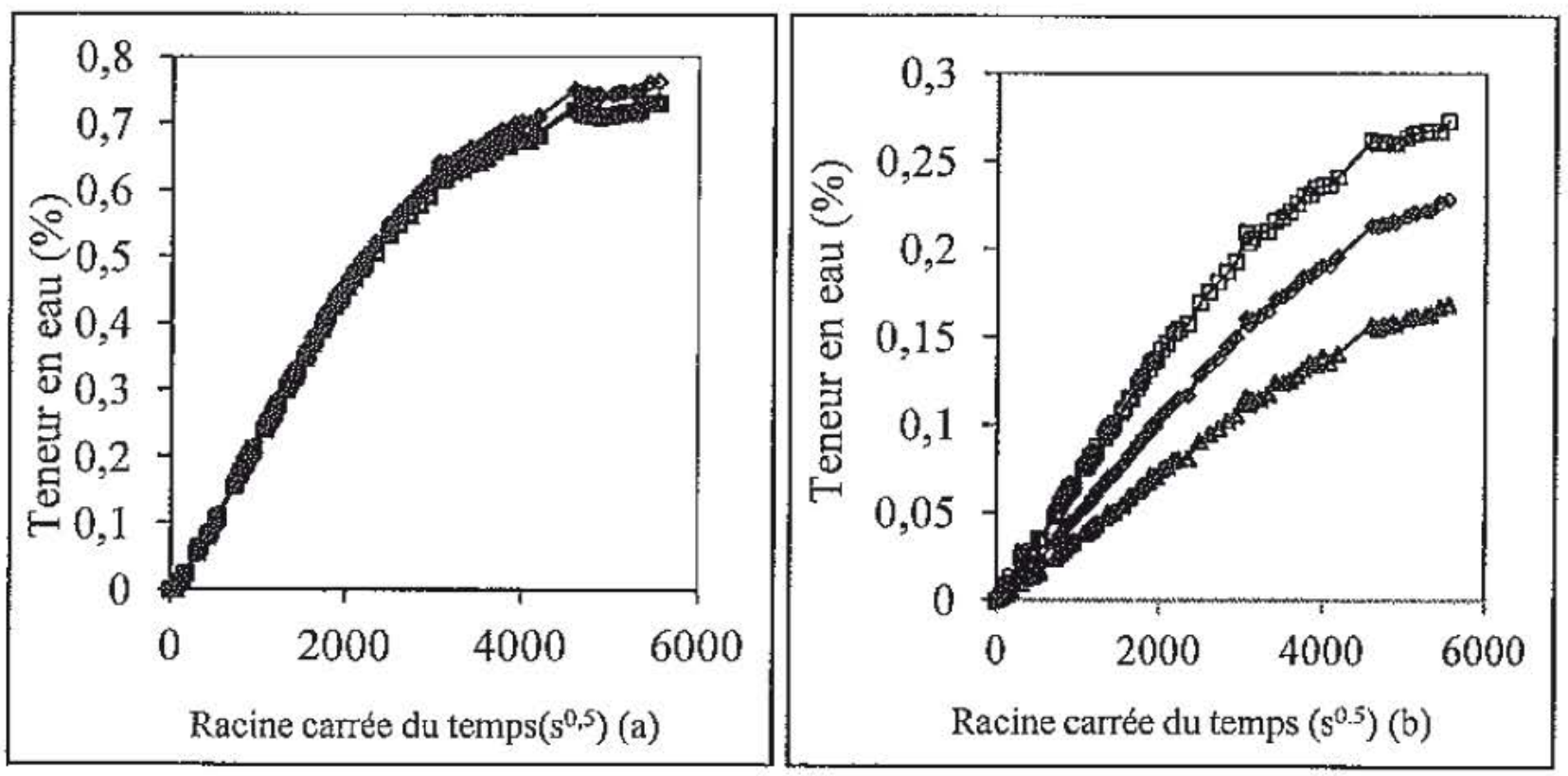

Figure 2. a) Courbes de suivi gravimétrique des trois échantillons de résine pure;

b) Courbes de suivi gravimétrique des échantillons composites I (carrés),

5 (losanges) et 8 (triangles) 
La figure 2a représente les courbes de suivi gravimétrique des trois échantillons de résine pure, tandis que la figure $2 \mathrm{~b}$ représente les courbes de suivi gravimétrique des échantillons composites $n^{\circ} 1, n^{\circ} 5$ et $n^{\circ} 8$ contenant respectivement $41,6 \%$, $47,2 \%$ et $53,4 \%$ de fibres. La figure 2 a montre une bonne reproductibilité du phénomène de reprise en eau des échantillons de résine pure. Celle-ci présente une loi de comportement diffusif d'allure fickienne. On constate que les trois échantillons composites (figure $2 \mathrm{~b}$ ) présentent une évolution linéaire pendant plusieurs mois, jusqu'à ce qu'un palier indiquant la saturation du processus de diffusion, soit atteint. On remarque que la capacité maximale d'absorption d'humidité décroît significativement quand le taux de fibres augmente.

\subsubsection{Principe d'identification des paramètres de diffusion}

Les courbes de suivi gravimétrique des éprouvettes composites et de résine pure présentent une évolution linéaire et un palier indiquant la saturation du processus de diffusion, caractéristiques d'un comportement fickien. La loi de Fick (5) permet de prédire la distribution de l'humidité en fonction du temps et de l'espace :

$$
\frac{\partial c}{\partial \mathrm{t}}=\operatorname{div}(-\mathrm{Dgrad} \mathrm{c})
$$

où $\mathrm{c}$ est la teneur en eau et $\mathrm{D}$ le coefficient de diffusion.

Pour une plaque épaisse orthotrope, en intégrant la solution tridimensionnelle de l'équation (5) sur le volume (les dimensions $\mathrm{L}$, $\mathrm{l}$, et e sont respectivement la longueur, la largeur et l'épaisseur de la plaque), on peut déterminer la teneur en eau $\mathrm{M}(\mathrm{t})$ absorbée par la plaque (6) :

$$
\begin{aligned}
& \frac{\mathrm{M}}{\mathrm{M}_{\infty}}=\left(1-\left(\frac{8}{\pi^{2}}\right)^{3} \sum_{\mathrm{n}=0}^{\infty} \sum_{\mathrm{j}=0}^{\infty} \sum_{\mathrm{k}=0}^{\infty} \frac{1}{((2 \mathrm{i}+1)(2 \mathrm{j}+1)(2 \mathrm{k}+1))^{2}}\right) \times \exp \left(-\pi^{2} \mathrm{t}\left(\mathrm{D}_{1}\left(\frac{2 \mathrm{i}+1}{\mathrm{~L}}\right)^{2}+\right.\right. \\
& \left.\left.\mathrm{D}_{2}\left(\frac{2 \mathrm{j}+1}{\mathrm{l}}\right)^{2}+\mathrm{D}_{3}\left(\frac{2 \mathrm{k}+1}{\mathrm{e}}\right)^{2}\right)\right)
\end{aligned}
$$

où $M_{\infty}$ est la teneur en eau à saturation $D_{1}, D_{2}$ et $D_{3}$ sont les coefficients de diffusion dans les directions $\mathrm{x}$, $\mathrm{y}$ et $\mathrm{z}$.

On identifie les valeurs des coefficients de diffusion et de la teneur en humidité à saturation en minimisant l'écart quadratique q (7) entre les teneurs en humidité prédites par la solution analytique (6) et celles déduites des relevés expérimentaux:

$$
\mathrm{q}=\sum_{\mathrm{i}}\left[\mathrm{M}\left(\mathrm{t}_{\mathrm{i}}\right)-\mathrm{M}_{\mathrm{i}}\right]^{2}
$$

\subsubsection{Méthode d'identification}

Kondo et Taki (1984) ont montré que le coefficient de diffusion d'humidité d'un composite unidirectionnel dans la direction parallèle à l'axe des fibres est égal à celui de la résine pure $\left(D_{1}=D_{r}\right)$. Nous avons identifié le coefficient de diffusion transverse $\left(\mathrm{D}_{2}\right)$ et la capacité maximale d'absorption d'humidité (Ms) sur la base de 
cette hypothèse. La moyenne des valeur's déduites de l'analyse des 5 échantillons de résine pure $\left(5,23 \times 10^{-7} \mathrm{~mm}^{2} / \mathrm{s}\right)$ a été considérée en tant que coefficient de diffusion longitudinal $\left(\mathrm{D}_{\mathrm{I}}\right)$ pour les échantillons composites. La capacité maximale d'absorption d'humidité de la résine pure, obtenue par cette méthode est de $0,73 \%$. La relation (5) fournit la capacité maximale d'absorption d'humidité d'un composite. Cette étape est indépendante de l'identification du coefficient de diffusion transverse $\left(\mathrm{D}_{2}\right)$ à partir des équations (6-7) (cf. tableau 2$)$.

Tableau 2. Paramètres de diffusion d'humidité obtenus par identification

\begin{tabular}{|l|c|c|c|c|c|c|c|c|}
\hline $\begin{array}{l}\mathrm{N}^{\circ} \text { de } \\
1^{\prime} \text { échantillon }\end{array}$ & 1 & 2 & 3 & 4 & 5 & 6 & 7 & 8 \\
\hline $\mathrm{M}_{\infty}(\%)$ & $0,29 \pm$ & $0,30 \pm$ & $0,30 \pm$ & $0,26 \pm$ & $0,25 \pm$ & $0,24 \pm$ & $0,22 \pm$ & $0,21 \pm$ \\
& 0,01 & 0,01 & 0,02 & 0,01 & 0,01 & 0,01 & 0,01 & 0,01 \\
\hline $\mathrm{D}_{2}^{*} 10^{7}$ & $2,85 \pm$ & $3,21 \pm$ & $2,22 \pm$ & $2,21 \pm$ & $2,11 \pm$ & $1,96 \pm$ & $1,74 \pm$ & $1,40 \pm$ \\
$\left(\mathrm{mm}^{2} / \mathrm{s}\right)$ & 0,05 & 0,07 & 0,07 & 0,04 & 0,06 & 0,04 & 0,04 & 0,04 \\
\hline
\end{tabular}

\subsection{Comparaison des résultats identifiés avec les prédictions de modèles de transition d'échelles}

Les modèles de transition d'échelles permettent de prédire le comportement diffusif effectif à partir de ceux des constituants. Dans la présente étude, différentes relations de transition d'échelles sont considérées afin de déterminer la diffusivité transverse effective des échantillons composites unidirectionnels. Les relations fondamentales de ces modèles sont récapitulées dans le tableau 3.

Tableau 3. Relations d'homogénéisation pour les coefficients de diffusion effectifs

\begin{tabular}{|c|c|}
\hline Méthodes & Coefficient de diffusion effectif \\
\hline \multirow{2}{*}{$\begin{array}{l}\text { Halpin-Tsai } \\
\text { (Halpin et Kardos, } \\
\text { 1976) }\end{array}$} & $D_{1}=v_{f} D_{f}+\left(1-v_{f}\right) D_{r}=\left(1-v_{f}\right) D_{r}$ \\
\hline & $\mathrm{D}_{2}=\mathrm{D}_{\mathrm{r}}\left(\frac{1}{1+\mathrm{v}_{\mathrm{f}}}\right) ; \mathrm{D}_{\mathrm{f}}=0$ \\
\hline $\begin{array}{c}\text { Shen - Springer } \\
(1976)\end{array}$ & $\mathrm{D}_{2}=\mathrm{D}_{\mathrm{r}}\left(1-2 \sqrt{\frac{\mathrm{V}_{\mathrm{f}}}{\pi}}\right)$ \\
\hline $\begin{array}{l}\text { Springer - Tsai } \\
\text { (1967) }\end{array}$ & $D_{2}=D_{r}\left(1-2 \sqrt{\frac{v_{f}}{\pi}}\right)\left(1-v_{f}\right)^{-1}$ \\
\hline $\begin{array}{l}\text { Shirrel - Halpin } \\
(1977)\end{array}$ & $\mathrm{D}_{2}=\mathrm{D}_{r}\left(\frac{1}{1+\mathrm{v}_{\mathrm{f}}}\right)$ \\
\hline $\begin{array}{c}\text { Rayleigh } \\
(1892)\end{array}$ & $D_{2}=D_{r} \frac{1-v_{f}-0,3058 v_{f}^{4}}{\left(1+v_{f}-0,3058 v_{f}^{4}\right)\left(1-v_{f}\right)}$ \\
\hline $\begin{array}{l}\text { Woo- Piggott } \\
\qquad(1988)\end{array}$ & $\mathrm{D}_{2}=\frac{\mathrm{D}_{\mathrm{r}}}{1-\mathrm{v}_{\mathrm{f}}}\left[\frac{2}{\sqrt{1-\frac{4 \mathrm{v}_{\mathrm{f}}}{\pi}}} * \tan ^{-1} \sqrt{\frac{1+2 \sqrt{\frac{v_{\mathrm{f}}}{\pi}}}{1-2 \sqrt{\frac{v_{f}}{\pi}}}}-\frac{\pi}{2}+1-2 \sqrt{\frac{\mathrm{v}_{\mathrm{f}}}{\pi}}\right]$ \\
\hline
\end{tabular}




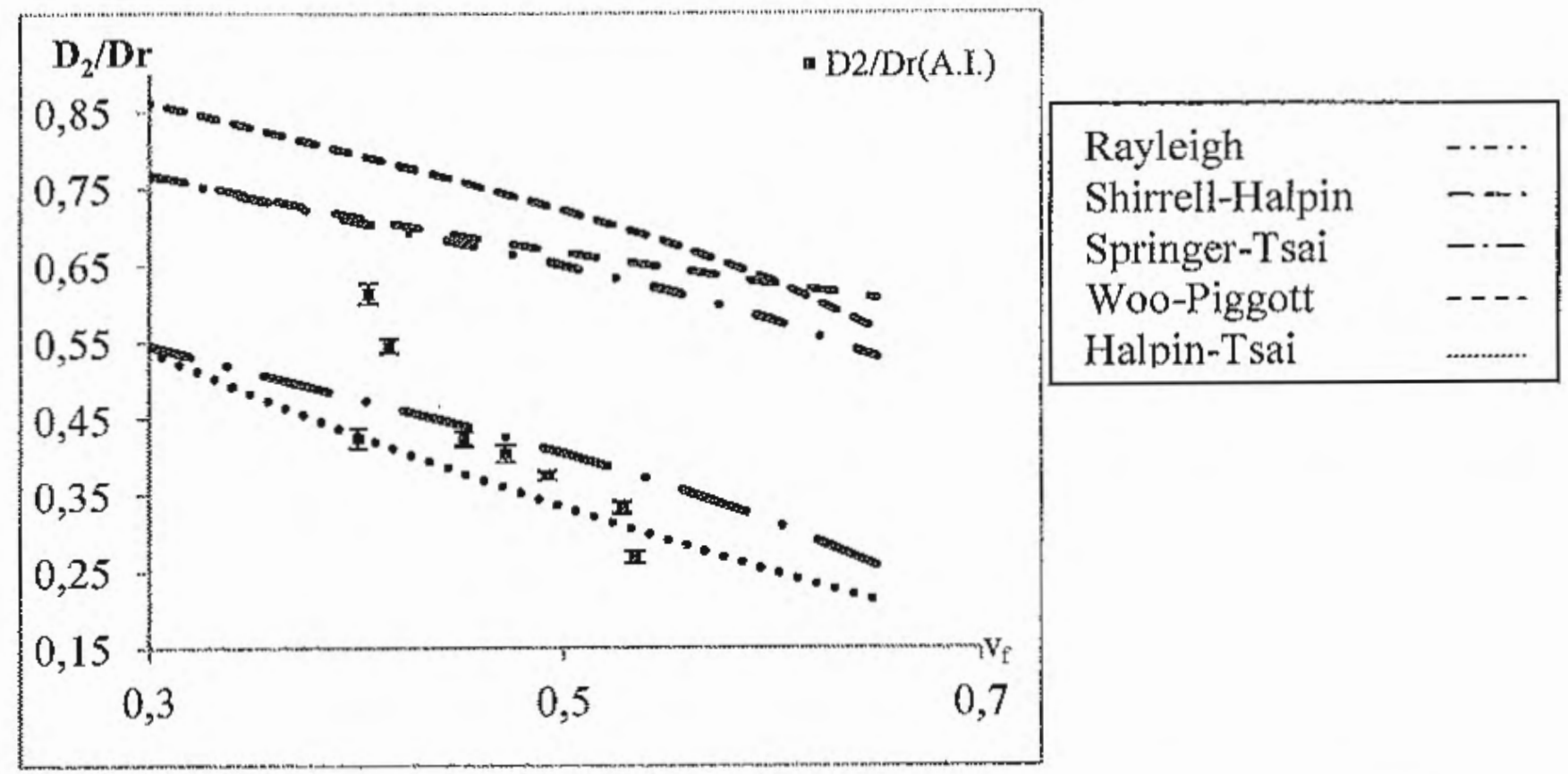

Figure 3. Comparaison des coefficients de diffusion transverse identifiés expérimentalement avec les valeurs prédites par les méthodes d'homogénéisation

La figure 3 montre les évolutions, en fonction de la fraction volumique de fibres, des coefficients de diffusion transverse déduits des modèles de transition d'échelles présentés dans le tableau 3 superposées aux résultats identifiés sur nos échantillons. D'après la figure 3 , les valeurs déduites des données expérimentales sont cohérentes avec les prédictions des modèles de Halpin-Tsai et de Springer-Tsai, tandis qu'elles s'écartent significativement des évolutions obtenues par l'intermédiaire des approches proposées par Rayleigh, Woo et Pigott ou encore de Shirell et Halpin.

\section{Identification des coefficients de dilatation hygroscopiques de composites à matrice polymère}

\subsection{Introduction - intérêt des capteurs à réseaux de Bragg}

Le développement des mesures de champs de déformations locaux au moyen de capteurs à réseaux de Bragg insérés au cœur de structures composites devrait fournir des données expérimentales à confronter aux prédictions des modèles de calcul (Mulle et al., 2007). Depuis quelques années, ces capteurs sont utilisés pour mesurer l'humidité relative (Kronenberg et al., 2002).

Le principe de fonctionnement des réseaux de Bragg a été présenté dans (Rao, 1997). Un réseau de Bragg se comporte comme un miroir pour une bande spectrale très fine autour de la longueur d'onde de Bragg $\lambda_{B}$ et reste transparent pour toutes les autres. $\lambda_{\mathrm{B}}$ est définie par: $\lambda_{\mathrm{B}}=2 n p$, où $n$ est l'indice de réfraction de la fibre optique et $p$ le pas du réseau. En pratique, ce pas fonctionne comme une jauge de déformation, traduisant la déformation (axiale) du réseau de Bragg lui-même. Une 
sollicitation hygrothermomécanique produit une variation de la longueur d'onde de Bragg. Dans le cas où le réseau de Bragg est inséré dans une structure composite, sa déformation est reliée à la déformation macroscopique. L'étude présentée ci-après consiste à exploiter ce phénomène pour déterminer le coefficient de dilatation hygroscopique d'un pli composite unidirectionnel.

\section{2. Élaboration et caractérisation des échantillons}

\subsection{1. Élaboration des échantillons}

Les échantillons étudiés sont des plaques composites constituées de fibres de verre et de résine polyester POLYLITE 420-73, fabriquées suivant la technique d'infusion de résine sous vide (VARI: Vacuum Assisted Resin Infusion). Les dimensions des plaques sont $90 \mathrm{~mm} \times 90 \mathrm{~mm} \times 2 \mathrm{~mm}$. Ces éprouvettes sont instrumentées par des fibres à réseaux de Bragg monomode X-FIBER (B), insérées à cour soit parallèlement aux fibres de renfort, soit perpendiculairement.

\subsubsection{Caractérisation des échantillons}

Les échantillons ont été immergés dans de l'eau distillée, à température ambiante. La cinétique de diffusion des échantillons a été caractérisée par suivi gravimétrique. Les déformations internes ont été analysées par l'intermédiaire du suivi du déplacement du pic de Bragg. La source utilisée est une source de largeur spectrale $30 \mathrm{~nm}$ centrée sur $1550 \mathrm{~nm}$. Le signal réfléchi est récupéré à l'aide d'un coupleur SMF 28 de JDC Uniphase. Ce signal est traité par un analyseur de spectre Anritsu MS9710B de résolution $70 \mathrm{pm}$. Les courbes obtenues en combinant les résultats de suivi gravimétrique et de déformations mesurées dans la direction transverse aux fibres de renfort sont représentées sur la figure 4.

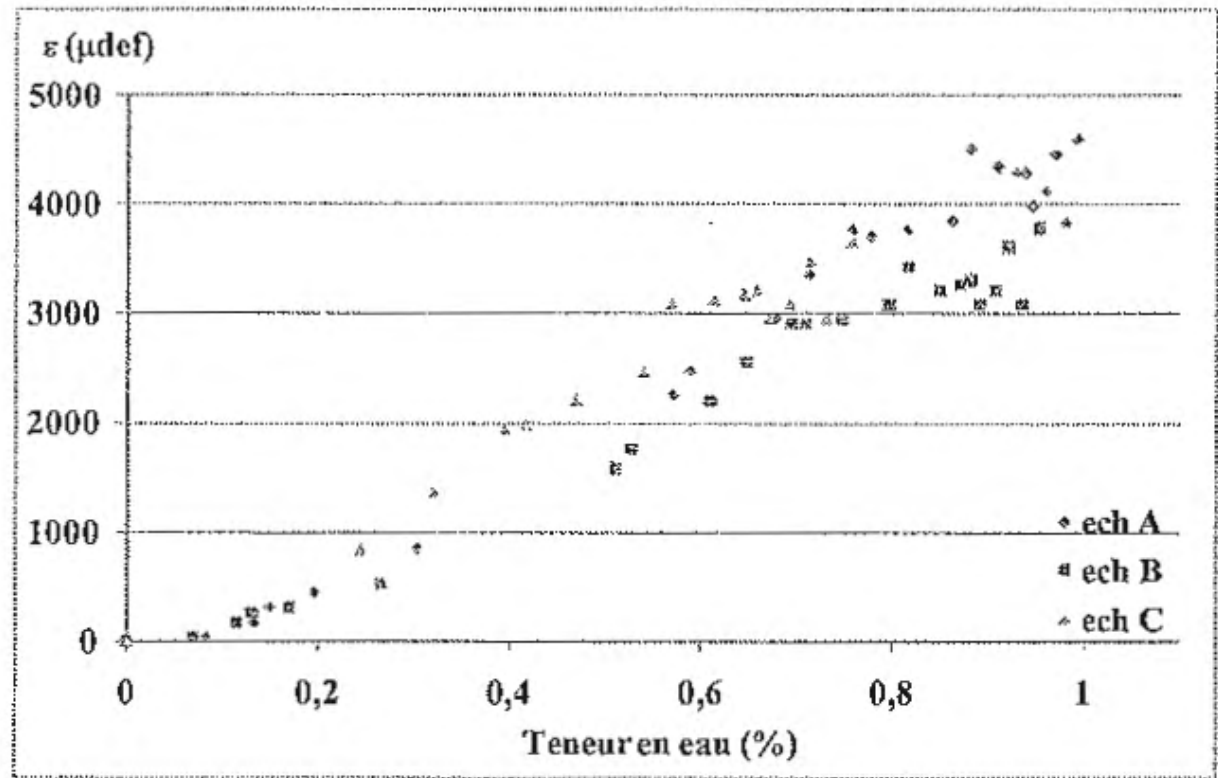

Figure 4. Évolution de la déformation transverse mesurée dans les échantillons composites, en fonction de la teneur en eau macroscopique 
A partir de l'évolution quasi linéaire de la déformation mesurée en fonction de la teneur en eau, on peut identifier, sur la base de la relation (2), le coefficient de dilatation hygroscopique macroscopique, dont la valeur moyenne est $\beta^{\mathrm{I}}=0,44$.

\section{Couplage diffusion d'humidité-contraintes internes}

La littérature fait état d'un grand nombre de phénomènes hygromécaniques couplés dans les matériaux polymères. Les effets induits par la plastification de ces derniers sous l'action de l'eau, qui auraient pourtant leur place dans cette section de l'article, ne seront pas présentés ici. Le lecteur intéressé pourra néanmoins se référer aux travaux de Youssef et al. (2009a). Nous nous limitons ici, dans un premier temps aux moditications des propriétés diffusives dues aux champs mécaniques, qu'ils soient externes ou internes. Dans un deuxième temps, nous nous consacrons aux phénomènes de couplage conduisant à des modifications des lois de sorption.

\subsection{Couplage diffusion d'humidité-états mécaniques multi-échelles : la théorie du volume libre}

Les travaux de Autran et al., (2002) rapportent une évolution des coefficients de diffusion et de la capacité maximale d'absorption d'humidité de composites en fonction de l'intensité d'un chargement extérieur appliqué à ces derniers. Ce type de comportement couplé, en l'absence d'anomalies de sorption, peut être modélisé par la théorie du volume libre. Une méthode de calcul de la variation du volume libre en fonction de l'état mécanique a été suggérée par Fahmy et Hurt (1980). En supposant le coefficient de diffusion fickien relié au volume libre par l'équation de Doolittle (1951), Neumann et Marom (1985) proposèrent l'expression suivante :

$$
\operatorname{Ln}\left(\frac{D_{\varepsilon}^{m}}{D_{0}^{m}}\right)=\frac{a}{v^{m}} \operatorname{Tr}\left(\varepsilon^{m}\right)\left(v_{f o}^{m}\left[v_{f v}^{m}+\operatorname{Tr}\left(\varepsilon^{m}\right)\right]\right)^{-1}
$$

oủ $D_{0}^{m}$ et $D_{E}^{m}$ sont les coefficients de diffusion pour la matrice libre de déformations et la matrice déformée, respectivement; a est un paramètre intrinsèque du matériau. $\mathrm{v}^{\mathrm{m}}$ est la fraction volumique de matrice dans le pli composite, $\mathrm{v}_{\mathrm{fo}}^{\mathrm{m}}$ est la fraction volumique de volume libre de la matrice époxy libre de déformations.

Dans la même série de travaux, on rapporte une évolution linéaire de la capacité maximale d'absorption d'humidité avec la trace de la déformation de la matrice :

$$
M_{\infty \varepsilon}^{\mathrm{m}}=M_{\infty 00}^{\mathrm{m}}+\operatorname{Tr}\left(\varepsilon^{\mathrm{ml}}\right) \times \frac{\rho^{\mathrm{w}}}{\rho^{\mathrm{m}}}
$$

Cette approche a permis de reproduire certaines données expérimentales (Autran et al., 2002). Elle a également été mise en ouvre dans le cadre d'une démarche multi-échelle dédiée à l'étude de composites carbone époxy (Youssef et al., 2009b). 
La figure 5 montre l'évolution de la teneur en eau, estimée par l'approche couplée. Deux empilements sont considérés : i) un stratifié croisé $\left[+55^{\circ} /-55^{\circ}\right]$, et ii) un unidirectionnel. La teneur atteinte à saturation pour l'unidirectionnel est de 1,5\%. Pour le stratifié croisé, on atteint seulement, en régime permanent, une valeur de $1,37 \%$. Ceci est dû à l'effet de l'empilement sur les profils d'états mécaniques multi-échelles. L'approche couplée génère des discontinuités aux interplis, en régime transitoire, qui n'existent pas dans les approches découplées. Ces discontinuités sont liées aux hétérogénéités d'états mécaniques d'un pli à l'autre.

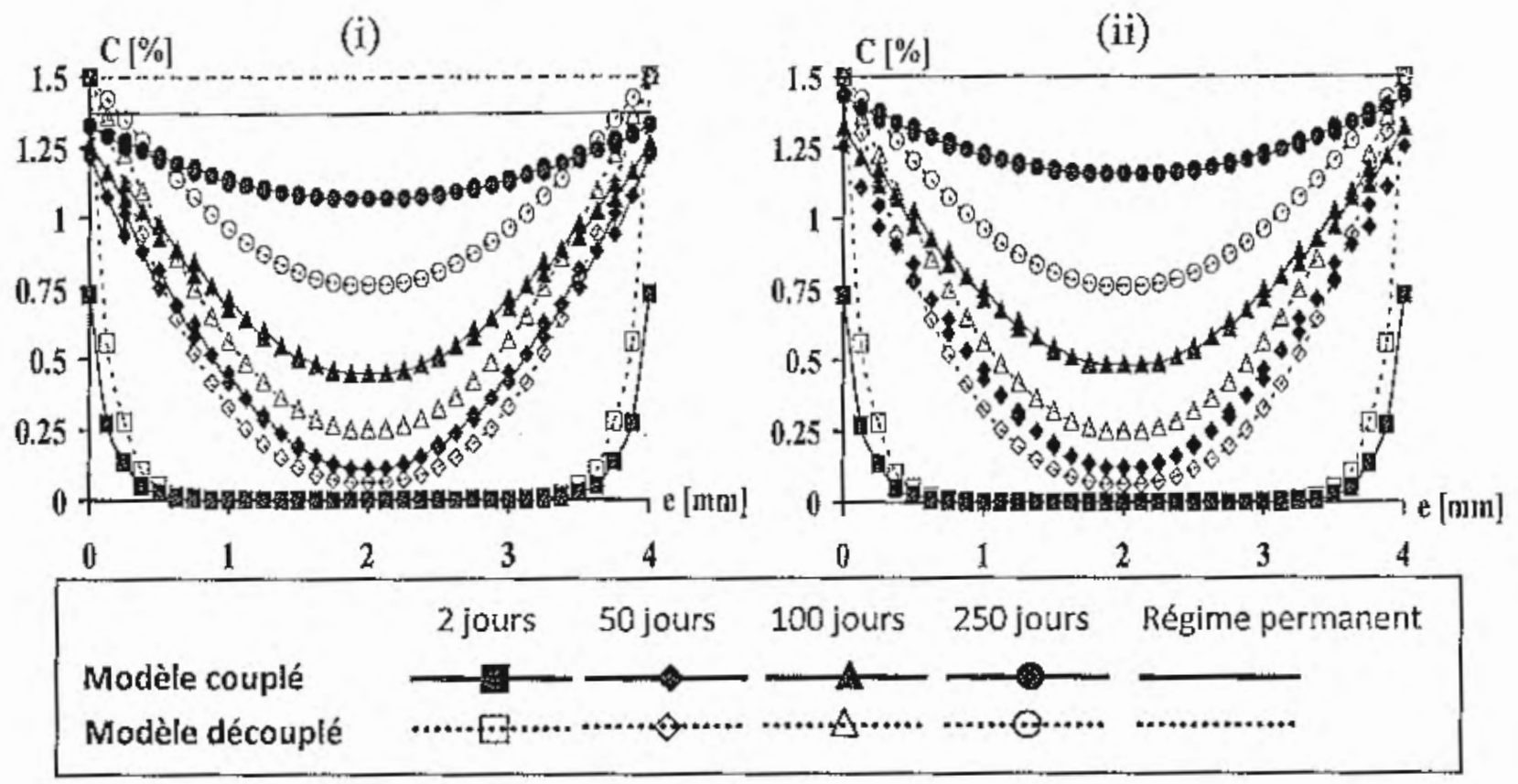

Figure 5. Profils de teneur en eau (i) pour un stratifié $\pm 55^{\circ}$,

(ii) pour un unidirectionnel

\subsection{Couplage diffusion d'humidité-états mécaniques multi-échelles : approches thermodynamiques et modifications des lois de sorption}

Derrien et Gilormini (2009) abordent le couplage diffusion d'humidité/états mécaniques par une approche thermodynamique basée sur la définition du potentiel chimique de l'eau $\widetilde{\mu}_{\mathrm{e}} \cdot \widetilde{\mu}_{\mathrm{e}}$ est la dérivée partielle de l'énergie libre $\mathrm{F}$, par rapport à la quantité d'eau $n_{e}$, cette dernière variable pouvant être reliée à la teneur en eau $C$ :

$$
\widetilde{\mu}_{\mathrm{e}}=\frac{\partial \mathrm{F}}{\partial \mathrm{n}_{\mathrm{e}}}=\frac{\partial \mathrm{F}}{\partial \mathrm{C}} \frac{\partial \mathrm{C}}{\partial \mathrm{n}_{\mathrm{e}}}
$$

L'équation (11) permet d'exprimer la cinétique de diffusion. Pour une diffusion unidirectionnelle, dans un polymère dont on néglige la variation de la masse volumique due à la déformation, la variation temporelle de la teneur en eau satisfait :

$$
\frac{\partial \mathrm{C}}{\partial \mathrm{t}}=\mathrm{D}\left[\left(1+\alpha \eta^{2} \mathrm{C}\right) \frac{\partial^{2} \mathrm{C}}{\partial \mathrm{x}^{2}}+\alpha\left(\frac{\partial \mathrm{C}}{\partial \mathrm{x}}\right)^{2}\right] \text { où } \alpha=\frac{2 \mathrm{~A}_{0} \mathrm{E}_{\mathrm{p}}}{3\left(1-v_{\mathrm{p}}\right)} \text { et } \mathrm{A}_{0}=\frac{3 \omega_{\mathrm{e}}}{\mathrm{RT} \rho_{0}}
$$


où $\mathrm{D}, \mathrm{E}_{\mathrm{p}}, v_{\mathrm{p}}$ et $\eta$ sont, respectivement, le coefficient de diffusion, le module de Young, le coefficient de Poisson et le coefficient de dilatation hygroscopique du polymère. $\rho_{0}$ est la masse volumique du polymère à l'état libre de déformations et $\omega_{\mathrm{e}}$ la masse molaire de l'eau. Ce modèle (Durier, 2008) suppose la masse volumique du polymère constante, ce qui est inexact. Ainsi, le volume $V_{p}$ du polymère s'exprime en fonction du volume $V_{0}$ du matériau libre de déformations et de la trace du tenseur des déformations $\varepsilon_{\mathrm{p}}$ comme : $\mathrm{V}_{\mathrm{p}}=\mathrm{V}_{\mathrm{o}}\left(\operatorname{Tr} \varepsilon_{\mathrm{p}}+1\right)$. La masse volumique $\rho_{\mathrm{p}}$ du polymère déformé s'écrit en fonction de $\rho_{0}$, en se basant sur la conservation de la quantité de matière (et donc de la masse) du matériau, au cours du processus de diffusion :

$$
\rho_{\mathrm{p}}=\frac{\mathrm{m}_{\mathrm{p}}}{\mathrm{V}_{\mathrm{p}}}=\frac{\mathrm{m}_{0}}{\mathrm{~V}_{\mathrm{p}}}=\rho_{0} \frac{1}{\left(\operatorname{Tr} \varepsilon_{\mathrm{p}}+1\right)}
$$

Dans le cas où la dépendance de la masse volumique du polymère, vis-à-vis de la teneur en eau, est prise en compte pour établir la loi de diffusion, on obtient :

$$
\frac{\partial \mathrm{C}}{\partial \mathrm{t}}=\mathrm{D}\left[\left(1+\alpha_{1} \eta^{2} \mathrm{C}+\alpha_{2} \eta^{3} \mathrm{C}^{2}\right) \frac{\partial^{2} \mathrm{C}}{\partial \mathrm{x}^{2}}+\eta^{2}\left(\alpha_{1}+\alpha_{3} \mathrm{C}\right)\left(\frac{\partial \mathrm{C}}{\partial \mathrm{x}}\right)^{2}\right]
$$

où, $\alpha_{1}=-3 \mathrm{~A}_{0} \mathrm{k}_{\mathrm{p}} \operatorname{Tr}\left(\varepsilon_{\mathrm{p}}\right)+2 \alpha \operatorname{Tr}\left(\varepsilon_{\mathrm{p}}\right)+\alpha, \alpha_{2}=9 \mathrm{~A}_{0} \mathrm{k}_{\mathrm{p}}-3 \alpha, \alpha_{3}=\eta \alpha_{2}-\frac{2 \eta \alpha^{2}}{\mathrm{~A}_{0} \mathrm{k}_{\mathrm{p}}}$.

Les équations (11) et (13) permettent de considérer des lois de diffusion qui diffèrent des modèles classiques rencontrés dans la littérature. La figure 6 présente les effets du coefficient de dilatation hygroscopique ainsi que de l'application d'une pression hydrostatique sur la teneur en eau globale.
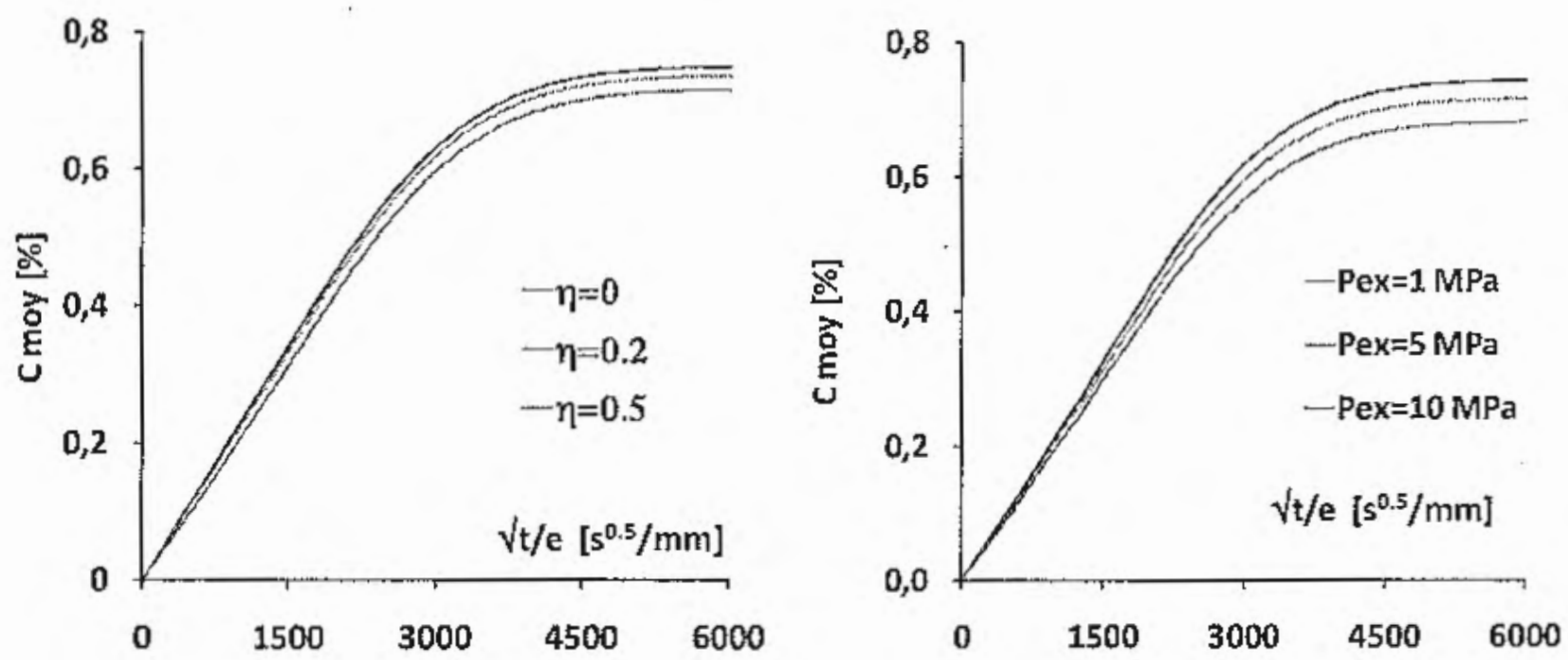

Figure 6. Effets du coefficient de dilatation et d'une pression additionnelle sur la teneur en eau 


\section{Conclusion}

Ce travail dresse les contours d'une étude sur les effets de l'environnement sur la durabilité des composites. Les essais de vieillissement permettent de caractériser le comportement diffusif. Dans un cadre numérique multi-échelle, deux types d'approches peuvent être menées. La première consiste à dissocier le calcul des teneurs en eau et des contraintes internes. Dans la seconde, la détermination de ces grandeurs repose sur des approches couplées. Concernant cette dernière approche, deux modèles basés, pour l'un, sur la théorie du volume libre et, pour l'autre, sur l'écriture « thermodynamique » du potentiel chimique, ont été présentés.

\section{Bibliographie}

Allred R.E., Hall N.H. (1979). Volume fraction determination of Kevlar 49/Epoxy composites. Polymer engineering and science, vol. 19, p. 907-909.

Augl J.M. (1979). Moisture sorption and diffusion in Kevlar 49 and aramid fiber. NSWC/TR, p. $79-51$.

Autran M., Pauliard R., Gautier L., Mortaigne B., Mazeas F., Davies P. (2002), Influence of mechanical stresses on the hydrolytic aging of standard and low styrene unsaturated polyester composites. Journal of Applied Polymer Science, vol. 84, p. 2185-2195.

Carter H.G., Kibler K.G. (1978). Langmuir-Type Model for Anomalous Moisture Diffusion in Composite Resins. Journal of Composite Materials, vol. 12, p. 118-131.

Crank J. (1975). The mathematics of diffusion. Clarendon Press, Oxford.

Delasi R., Whiteside J.B. (1978). Effect of Moisture on epoxy resins and composites, Advanced Composite Materials - Environmental effects. ASTM STP 658.

Derrien K., Gilormini P. (2009). The effect of moisture-induced swelling on the absorption capacity of transversely isotropic elastic polymer-matrix composites. International Journal of Solids and Structures, vol. 46, p. 1547-1553.

Durier A.L. (2008). Contribution à l'étude de l'interaction contraintes-diffusion dans les polymères. Thèse de l'Ecole Nationale des Arts et Métiers de Paris, Paris.

Doolittle A. (1951). Studies in Newtonian flow. II. The dependence of the viscosity of liquids on free-space. Joumal of Applied Physics, vol. 22, p. 1471-1475.

Fahmy A., Hurt J.C. (1980). Stress Dependence of Water Diffusion in Epoxy Resin. Polymer Composites, vol. 1, p. 77-80.

Fick A. (1855). Ueber Diffusion. Annalen der Physik, vol. 170, p. 59-86.

Fréour S., Lacoste E., Fajoui J., Jacquemin F. (2011). On the meaning of the chosen setaveraging method within Eshelby-Kröner self-consistent scale transition model: the geometric mean versus the classical arithmetic average. Zeitschrift fiir Angewandte Mathematik und Mechanik/Joumal of Applied Mathematics and Mechanics, vol. 91, p. 689-698. 
Fréour S., Jacquemin F., Guillén, R. (2007). On the use of the geometric mean approximation in estimating the effective hygro-elastic behaviour of fiber-reinforced composites. Journal of Materials Science, vol. 42, p. 7537-7543.

Fréour S., Jacquemin F., Guillén R. (2005). On an analytical self-consistent model for internal stress prediction in fiber-reinforced composites submitted to hygroelastic load. Journal of Reinforced Plastics and Composites, vol. 24, p. 1365-1377.

Gopalan R., Rao R.M.V.G.K., Murthy M.V.V., Dattaguru B. (1986). Diffusion Studies on Advanced Fibre Hybrid Composites. Journal of Reinforced Plastics and Composites, vol. 5 , p. 51-61.

Green P. (1991). Fibers volume fraction determination of carbon-epoxy composites using an acid digestion bomb. Journal of materials science letters, vol. 10, p. 1162-1164.

Halpin J.C., Kardos J.L. (1976). The Halpin-Tsai equations: A review. Polymer Engineering and Science, vol.16, p. 344-352.

Jacquemin F., Fréour S., Guillén R. (2006). Analytical modeling of transient hygro-elastic stress concentration - Application to embedded optical fiber in a non-uniform transient strain field. Composites Science and Technology, vol. 66, p. 397-406.

Jacquemin F., Fréour S., Guillén R. (2005). A Hygro-Elastic Self-Consistent Model for FiberReinforced Composites. Journal of Reinforced Plastics and Composites, vol. 24, p. 485-502.

Jacquemin F., Vautrin A. (2002). A closed-form solution for the internal stresses in thick composite cylinders induced by cyclical environmental conditions. Composite Structures, vol. 58, p. 1-9.

Kondo K,, Taki T. (1984). Environmental effects on composite materials. Technomic Publishing.

Kronenberg P., Rastogi P.K., Giaccari P., Limberger H.G. (2002). Relative humidity sensor with optical fiber Bragg gratings. Optics Letters, vol, 27, p. 1385-1387.

Lacoste E., Fréour S., Jacquemin F. (2010). On the validity of the Kröner-Eshelby scale transition model for inclusion with various morphologies. Mechanics of Materials, vol. 42, p. 218-226.

Loos A.C., Springer G.S. (1979). Moisture absorption of graphite/epoxy composites immersed in liquids and in humid air. Journal of Composite Materials, vol. 13, p. 131-146.

Mulle M., Zitoune R., Collombet F., Grunevald Y.-H. (2007). Thermal expansion of carbonepoxy laminates measured with embedded FBGs - Comparison with other experimental techniques and numerical simulation. Composites Part A, vol. 38, p. 1414-1424.

Neumann S., Marom G. (1985). Stress Dependence of the Coefficient of Moisture Diffusion in Composite Materials. Polymer Composites, vol. 6, p. 9-12.

Pierron F., Poirette Y., Vautrin A. (2002). A Novel Procedure for Identification of 3D Moisture Diffusion Parameters on Thick Composites: Theory, Validation and Experimental Results. Joumal of Composite Materials, vol. 36, p. 2219-2243.

Rao Y.J. (1997). In-fibre Bragg grating sensors. Measurements Sciences and Technology, vol.8, p. 355-375. 
Rayleigh L. (1982). On the Instability of Cylindrical Fluid Surfaces. Philosophical Magazine, vol. 34, p. 177-180.

Shen C.H., Springer G.S. (1976). Moisture Absorption and Desorption of Composite Materials. Joumal of Composite Materials, vol. 10, p. 2-20.

Shirrell C.D. (1978). Diffusion of Water Vapor in Graphite/Epoxy Composites. ASTM, STP 658 .

Shirell C.D., Halpin J. (1977). Moisture Absorption and Desorption in Epoxy Composite Laminates. ASTM STP 617.

Springer G.S., Tsai S.W. (1967). Thermal conductivities of unidirectional materials. Journal of Composite Materials, vol. 1, p. 166-173.

Tsai S.W. (1987). Composite Design. Think Composites.

Woo M., Piggott M. (1988). Water Absorption of Resins and Composites: IV. Water Transport in Fiber Reinforced Plastics. Journal of Composites Technology and Research, vol. 10, p. 20-24.

Woo M., Piggott M. (1987). Water Absorption of Resins and Composites: I. Epoxy Homopolymers and Copolymers. Journal of Composite Technology Research, vol. 9, p. 101-107.

Ye B.S, Svenson A.L., Bank L.C. (1995). Mass and volume fraction properties of pultruded glass fibers-reinforced composites. Composites, vol. 26, p. 725-731.

Youssef G., Fréour S., Jacquemin F. (2009a). Effects of moisture dependent constituents properties on the hygroscopic stresses experienced by composite structures. Mechanics of Composite Materials, vol. 45, p. 369-380.

Youssef G., Fréour S., Jacquemin F. (2009b). Stress-dependent moisture diffusion in composite materials. Joumal of Composite Materials, vol. 43, p. 1621-1637. 